\title{
Delayed post-hypoxic leukoencephalopathy in an adult with COVID-19
}

\author{
Brannon L. Vines $^{1}$ (D) Shruti P. Agnihotri ${ }^{1}$
}

Received: 15 December 2020 / Revised: 2 April 2021 / Accepted: 14 April 2021 / Published online: 11 May 2021

(c) Journal of NeuroVirology, Inc. 2021

\begin{abstract}
As the novel coronavirus, SARS-CoV-2, has enveloped the world in a pandemic, it has become clear that the symptoms extend far beyond the respiratory system and have particularly caused a wide range of neurologic CNS complications, including diffuse leukoencephalopathy. Here, we describe a case of a 59-year-old male with severe COVID-19 infection who developed severe encephalopathy, which persisted well after his acute infection had subsided and had begun to improve from his respiratory dysfunction. He was found to have diffuse leukoencephalopathy with concomitant diffusion restriction on MR imaging. This case represents a delayed onset of leukoencephalopathy secondary to hypoxia in a small but growing cohort of COVID-related leukoencephalopathy due to similarities in imaging features and lack of superior alternate diagnosis. Patient's clinical improvement suggests reversibility with likely pathology being demyelination rather than infarction.
\end{abstract}

Keywords Coronavirus $\cdot$ SARS-CoV-2 $\cdot$ White matter $\cdot$ Encephalopathy $\cdot$ Demyelination

\section{Background}

At the start of the coronavirus disease 2019 (COVID-19) outbreak in December 2019, it was initially thought that the novel coronavirus, SARS-CoV-2, caused only typical but potentially severe respiratory symptoms. However, as the pandemic spread, it quickly became clear that the disease caused multi-system involvement including its effects on the kidneys, heart, and the brain (Gavriatopoulou et al. 2020; Koralnik and Tyler 2020). While neurologic symptoms have been common as part of the early disease presentation, severe neurologic complications such as encephalopathy, strokes, and seizures have been reported in patients with COVID-19 (Somani et al. 2020; Liotta et al. 2020; Helms et al. 2020). Availability of brain imaging is limited in many cases due to strict isolation precautions and severity of respiratory compromise. Cerebrospinal fluid (CSF) analyses in some patients show evidence of inflammation but SARS-CoV-2 has not been isolated in CSF from most of these cases (Farhadian et al. 2020). COVID19-associated encephalopathy is largely thought to be secondary to hypoxia and sequelae of multi-organ failure. However, there are few reports of leukoencephalopathy identified on

Brannon L. Vines

brannonvines@uabmc.edu

1 The University of Alabama, SC 440C, 1720 2nd Ave S, Birmingham, AL 35294, USA imaging or autopsy, which suggests the possibility of demyelination as an underlying pathology (Reichard et al. 2020)

Here, we present a case of delayed leukoencephalopathy with associated diffusion restriction and without microhemorrhages in a patient with COVID-19 after stabilization of his respiratory disease.

\section{Presentation}

A 59-year-old male with a past medical history of diabetes and hypertension with known COVID-19 exposure presented initially to a community hospital with cough, fever, chills, and shortness of breath of 4 days duration. Due to worsening respiratory status, he was transferred to a tertiary medical center the day after presentation for consideration of advanced therapies. He was immediately intubated on arrival due to hypoxia and respiratory compromise with an $\mathrm{O}_{2}$ saturation of $61 \%$. Shortly thereafter, SARS-CoV-2 was isolated from his nasopharyngeal swab. Treatment for the severe respiratory disease included prone position ventilation and a course of remdesivir. He was placed on continuous renal replacement therapy for acute kidney injury and there were no precipitous abnormalities in his electrolytes during his admission.

As sedation was weaned, patient was difficult to arouse. He responded to pain stimuli by minimal withdrawal in all limbs, so a CT scan of the head was obtained 2 weeks into 
Fig. 1 CT scan of the head is notable for absence of subcortical hypodensities

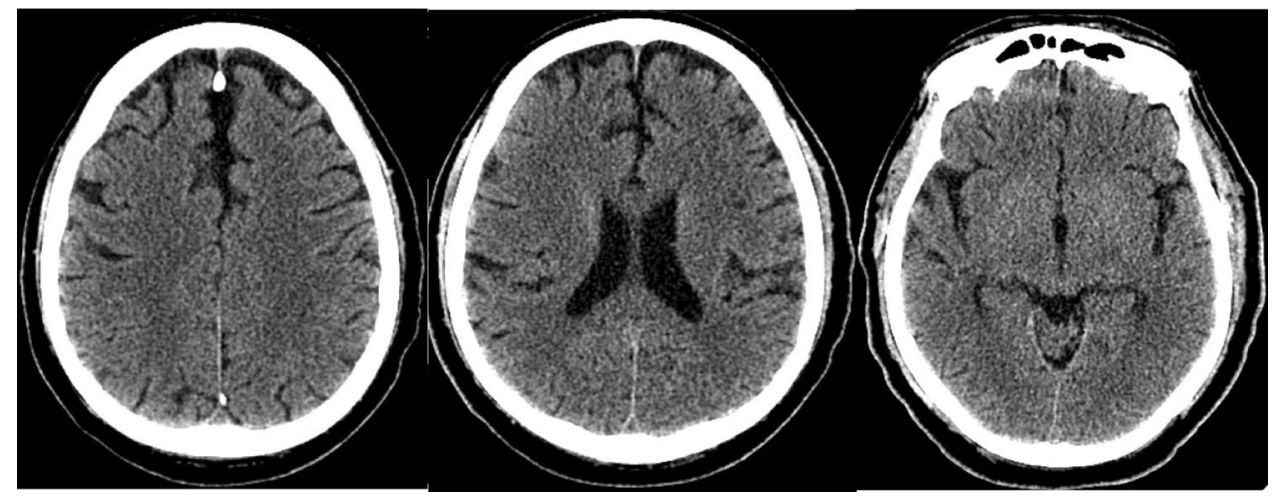

his hospitalization and was normal Fig. 1. Later, 1 month into his hospitalization, an MRI of the brain without gadolinium was performed for his persistent encephalopathy. MRI Fig. 2 revealed a diffuse confluent hyperintense signal of the subcortical white matter on T2/FLAIR sequence that spared U-fibers, cortex, and deep brain structures. Diffusion-weighted imaging revealed diffuse diffusion restriction throughout the subcortical white matter in the same pattern as the hyperintensity on T2/FLAIR. Susceptibility-weighted imaging did not show any hemorrhage.

Neurological examination demonstrated preserved cranial nerve reflexes, but no response to noxious stimuli. Lumbar puncture was performed revealing a white blood cell count of 1 , red blood cell count of 1 , glucose level of $79 \mathrm{mg} / \mathrm{dl}$, and protein level of $29 \mathrm{mg} / \mathrm{dl}$. CSF testing for culture and routine microbial stains, HSV PCR, VZV PCR, CMV PCR, AFB stains, and cryptococcal antigen were all negative. Additionally, SARS-CoV-2 PCR testing was also negative in CSF. Long-term video EEG monitoring was performed and revealed generalized background slowing and state-dependent triphasic waves, but no asymmetric or epileptiform features. Repeat MRI of the brain was performed 12 days after the initial scan Fig. 3 showing evolution of diffusion restriction. The T2 FLAIR hyperintensity in the white matter persisted. There was no abnormal contrast enhancement after gadolinium administration.

Over the next month, patient's mental status slowly improved to the point of being awake, alert, oriented, following simple commands, and mouthing a few words. He was then transferred to a long-term acute care where he was successfully weaned off the ventilator. His neurological status continued to gradually improve.

\section{Discussion}

Herein, we report a case of severe encephalopathy in the wake of severe COVID-19 infection. Prolonged encephalopathy in patients with severe COVID-19 is associated with increased mortality and poor outcomes (Liotta et al. 2020). This was noted in our patient as well, who survived and had neurologic improvement but required prolonged hospitalization. The findings of diffuse symmetric T2/FLAIR hyperintensities on MRI can be seen in many conditions including posterior reversible leukoencephalopathy syndrome (PRES), hereditary leukodystrophies, infectious causes like HIVassociated leukoencephalopathy, demyelinating disorders like acute disseminated encephalomyelitis (ADEM), and toxic-metabolic causes (Sarbu et al. 2016). In addition to the pattern of diffusion restriction and T2/FLAIR hyperintensity being dissimilar to relatively common presentations of atypical PRES, there was a lack of PRES risk factors in this case such as precipitous hypertension or commonly offending drugs like tacrolimus (McKinney et al. 2007). The normal CSF helps rule out alternate diagnoses such as active inflammatory or infectious processes as the cause of his leukoencephalopathy. Overall, the COVID-19-positive status of the patient, severe nature of the COVID-19 infection, lack of clear alternative diagnoses, and the similarity to previously reported cases of leukoencephalopathy in COVID19 support the diagnosis of leukoencephalopathy related to COVID-19 in this case. The radiological presentation is unique given the severity and diffuse nature of leukoencephalopathy without microhemorrhages, unlike those reported previously (Radmanesh et al. 2020). We also demonstrate the presence and evolution of diffusion restriction changes 4 weeks after hypoxia, with a normal CT scan of the head initially, suggesting a delayed mechanism of onset.

Many theories are proposed for possible etiologies of diffuse leukoencephalopathy with COVID-19. A toxicmetabolic cause including delayed response to hypoxemia is postulated to be the most likely cause (Lang et al. 2020). Our patient had severe hypoxemia leading to mechanical ventilation. Delayed post-hypoxic leukoencephalopathy is the most likely cause in our patient given the presence of diffusion restriction changes about 4 weeks after hypoxia. This is distinct due to the reversible nature secondary to demyelination (Katyal et al. 2018; Shprecher and Mehta 2010). An interval period of improved or normal neurocognitive 


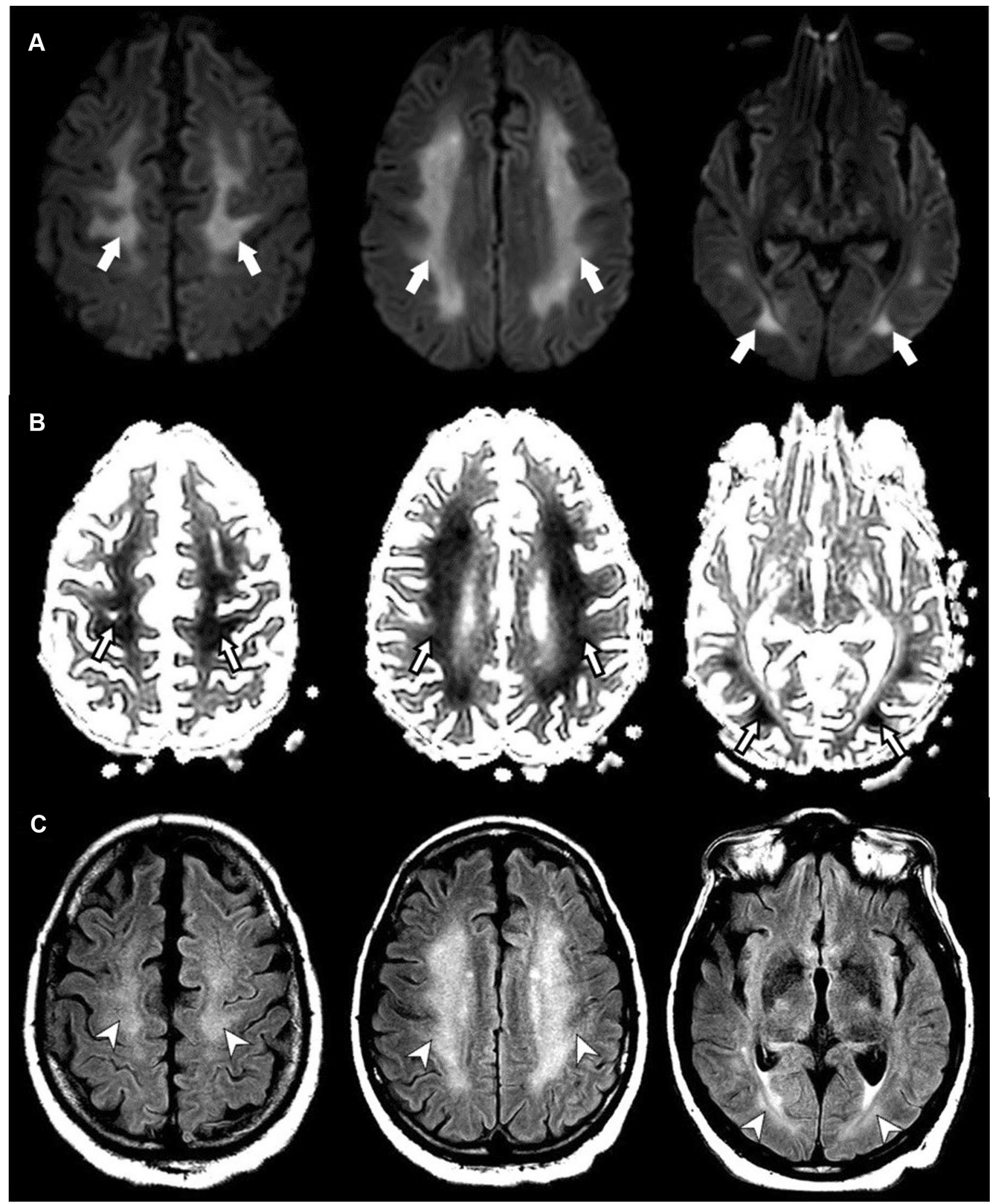

Fig. 2 a Diffusion-weighted imaging (DWI) and $\mathbf{b}$ apparent diffusion coefficient (ADC demonstrate corresponding hyperintensity on DWI and hypointensity on ADC which suggests diffuse confluent acute diffusion restriction of the subcortical white matter, sparing the U-fibers, cortex, and deep brain structures. c T2/FLAIR sequence that demonstrates diffuse confluent hyperintensity throughout the subcortical white matter, corresponding to the diffusion restriction, and also sparing U-fibers, cortex, and deep brain structures 


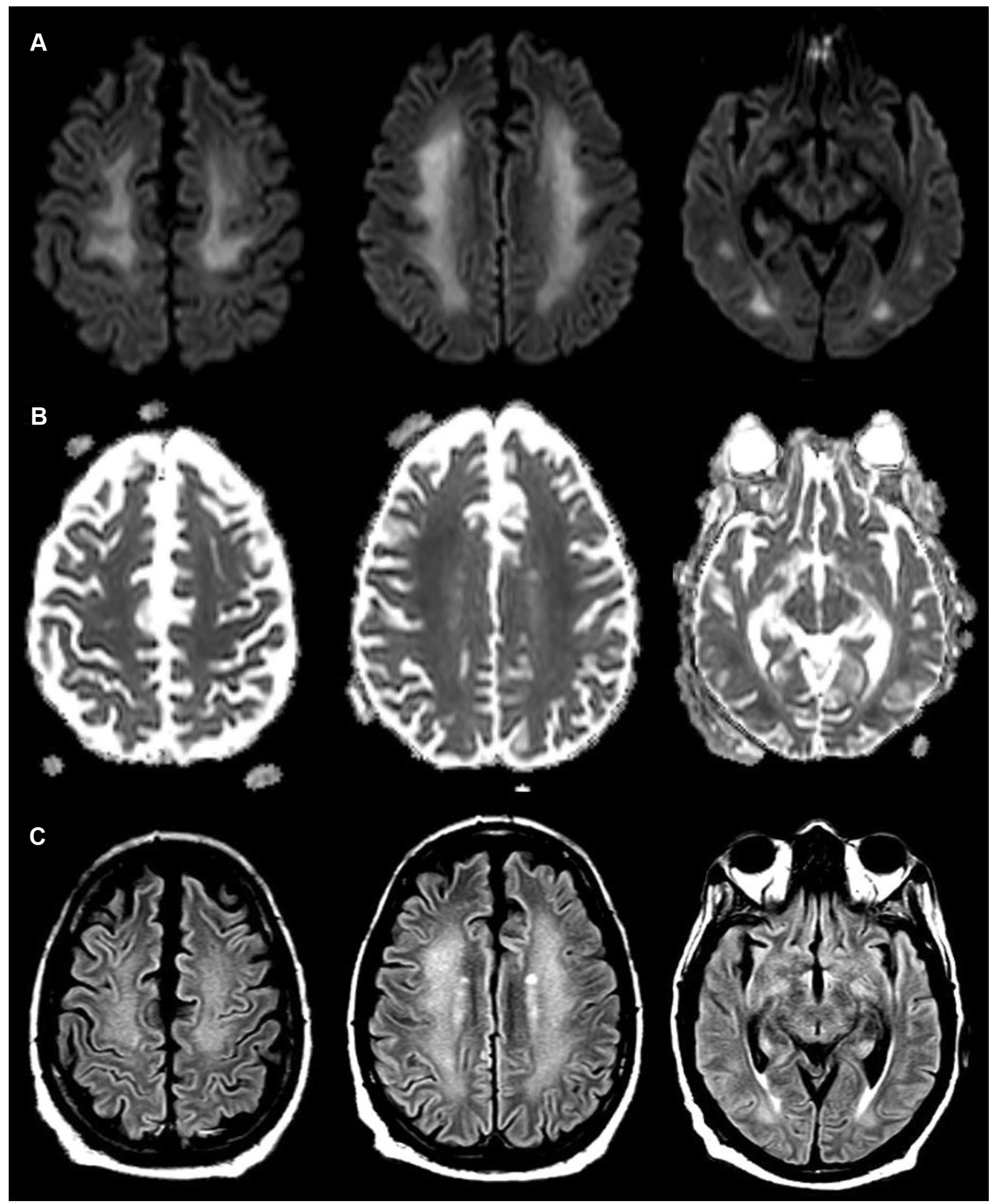

Fig. 3 a Diffusion-weighted imaging (DWI) and $\mathbf{b}$ apparent diffusion coefficient (ADC) show continued hyperintensity on DWI but evolution to isointensity on ADC which suggests subacute diffusion restric- tion with no interval development of additional diffusion restriction. c Diffuse confluent T2/FLAIR subcortical white matter hyperintensity without interval change 
status could not be established in our patient due to severe respiratory failure and possibly due to COVID-19-related encephalopathy. Alternatively, the sequela of direct neurotropism has been proposed. In our case, as in other case series, SARS-CoV-2 was not detected in CSF. Neuropathological studies in COVID-19 have demonstrated no direct damage to the CNS from the virus but changes secondary to hypoxia were noted (Solomon et al. 2020). Delayed post-hypoxic leukoencephalopathy should be considered in patients with prolonged encephalopathy following severe COVID-19, especially given its reversible disease course. Long-term follow-up of survivors, further imaging, and clinicopathologic correlations are needed to better characterize the leukoencephalopathy in these patients.

\section{References}

Farhadian S, Glick LR, Vogels CBF, Thomas J, Chiarella J, CasanovasMassana A et al (2020) Acute encephalopathy with elevated CSF inflammatory markers as the initial presentation of COVID-19. BMC Neurol 20(1):248

Gavriatopoulou M, Korompoki E, Fotiou D, Ntanasis-Stathopoulos I, Psaltopoulou T, Kastritis E et al (2020) Organ-specific manifestations of COVID-19 infection. Clin Exp Med 20(4):493-506

Helms J, Kremer S, Merdji H, Clere-Jehl R, Schenck M, Kummerlen C et al (2020) Neurologic features in severe SARS-CoV-2 infection. N Engl J Med 382(23):2268-2270

Katyal N, Narula N, George P, Nattanamai P, Newey CR, Beary JM (2018) Delayed post-hypoxic leukoencephalopathy: a case series and review of the literature. Cureus 10(4):e2481
Koralnik IJ, Tyler KL (2020) COVID-19: a global threat to the nervous system. Ann Neurol 88(1):1-11

Lang M, Buch K, Li MD, Mehan WA Jr, Lang AL, Leslie-Mazwi TM et al (2020) Leukoencephalopathy associated with severe COVID19 infection: sequela of hypoxemia? AJNR Am J Neuroradiol 41(9):1641-1645

Liotta EM, Batra A, Clark JR, Shlobin NA, Hoffman SC, Orban ZS et al (2020) Frequent neurologic manifestations and encephalopathyassociated morbidity in Covid-19 patients. Ann Clin Transl Neurol 7(11):2221-2230

McKinney AM, Short J, Truwit CL, McKinney ZJ, Kozak OS, SantaCruz KS et al (2007) Posterior reversible encephalopathy syndrome: incidence of atypical regions of involvement and imaging findings. AJR Am J Roentgenol 189(4):904-912

Radmanesh A, Derman A, Lui YW, Raz E, Loh JP, Hagiwara M et al (2020) COVID-19-associated diffuse leukoencephalopathy and microhemorrhages. Radiology 297(1):E223-E227

Reichard RR, Kashani KB, Boire NA, Constantopoulos E, Guo Y, Lucchinetti CF (2020) Neuropathology of COVID-19: a spectrum of vascular and acute disseminated encephalomyelitis (ADEM)like pathology. Acta Neuropathol 140(1):1-6

Sarbu N, Shih RY, Jones RV, Horkayne-Szakaly I, Oleaga L, Smirniotopoulos JG (2016) White matter diseases with radiologicpathologic correlation. Radiographics 36(5):1426-1447

Shprecher D, Mehta L (2010) The syndrome of delayed post-hypoxic leukoencephalopathy. NeuroRehabilitation 26(1):65-72

Solomon IH, Normandin E, Bhattacharyya S, Mukerji SS, Keller K, Ali AS et al (2020) Neuropathological features of Covid-19. N Engl J Med 383(10):989-992

Somani S, Pati S, Gaston T, Chitlangia A, Agnihotri S (2020) De novo status epilepticus in patients with COVID-19. Ann Clin Trans1 Neurol 7(7):1240-1244

Publisher's Note Springer Nature remains neutral with regard to jurisdictional claims in published maps and institutional affiliations. 